\title{
Far-infrared transmission in GaN, AIN, and AIGaN thin films grown by molecular beam epitaxy
}

\author{
J. Ibáñez, ${ }^{1}$ S. Hernández, ${ }^{1}$ E. Alarcón-Lladó, ${ }^{1}$ R. Cuscó, ${ }^{1}$ L. Artús, ${ }^{1, a)}$ S. V. Novikov, ${ }^{2}$ \\ C. T. Foxon, ${ }^{2}$ and E. Calleja ${ }^{3}$ \\ ${ }_{1}^{1}$ Institut Jaume Almera, Consell Superior d'Investigacions Científiques (CSIC), Lluís Solé i Sabarís s.n, \\ 08028 Barcelona, Catalonia, Spain \\ ${ }^{2}$ School of Physics and Astronomy, University of Nottingham, Nottingham NG7 2RD, United Kingdom \\ ${ }^{3}$ ISOM-Departamento Ingeniería Electrónica, ETSI Telecomunicación, Universidad Politécnica, \\ 28040 Madrid, Spain
}

(Received 3 April 2008; accepted 11 June 2008; published online 15 August 2008)

\begin{abstract}
We present a far-infrared transmission study on group-III nitride thin films. Cubic GaN and AIN layers and $c$-oriented wurtzite $\mathrm{GaN}, \mathrm{AlN}$, and $\mathrm{Al}_{x} \mathrm{Ga}_{1-x} \mathrm{~N}(x<0.3)$ layers were grown by molecular beam epitaxy on GaAs and $\mathrm{Si}(111)$ substrates, respectively. The Berreman effect allows us to observe simultaneously the transverse optic and the longitudinal optic phonons of both the cubic and the hexagonal films as transmission minima in the infrared spectra acquired with obliquely incident radiation. We discuss our results in terms of the relevant electromagnetic theory of infrared transmission in cubic and wurtzite thin films. We compare the infrared results with visible Raman-scattering measurements. In the case of films with low scattering volumes and/or low Raman efficiencies and also when the Raman signal of the substrate material obscures the weaker peaks from the nitride films, we find that the Berreman technique is particularly useful to complement Raman spectroscopy. (C) 2008 American Institute of Physics. [DOI: 10.1063/1.2968242]
\end{abstract}

\section{INTRODUCTION}

Group-III nitride compounds are currently being used to fabricate optoelectronic devices operating in the blue and near-ultraviolet (UV) regions as well as high-power microwave devices and field-effect transistors. Numerous studies have employed Raman scattering and far-infrared (IR) spectroscopies to investigate several physical properties of GaN, $\mathrm{AlN}$, and the ternary alloy AlGaN such as their phonon modes, the crystal quality and the orientation of the as-grown material, free-carrier effects, and the piezoelectric response. $^{1-18}$ It is now clear that the longitudinal optic (LO) phonons $\left[A_{1}(\mathrm{LO})\right.$ and $\left.E_{1}(\mathrm{LO})\right]$ of hexagonal AlGaN display a one-mode behavior, whereas the rest of the Raman or IR active phonon modes $\left[E_{2}, A_{1}(\mathrm{TO})\right.$ and $\left.E_{1}(\mathrm{TO})\right]$ exhibit a two-mode behavior. ${ }^{13,17}$ Similarly, it has been shown that the LO and transverse optic (TO) phonons of cubic AlGaN exhibit one-mode and two-mode behaviors, respectively. ${ }^{11}$

While visible Raman scattering is a highly sensitive technique for the study of the lattice dynamics in semiconductor alloys, many authors usually rely on IR reflectance to characterize the optical properties of group-III nitride crystals and structures. ${ }^{1,4-9,12-15}$ In contrast, only a few works reported IR transmission measurements, ${ }^{3,9,12,18}$ most of which were performed in normal-incidence configuration. Reports dealing with oblique-incidence IR transmission to study the phonons of group-III nitrides are scarce. ${ }^{3}$

As first demonstrated by Berreman, ${ }^{19}$ the frequency of both zone-center TO and LO phonons of polar materials can be directly measured in far-IR transmission experiments for radiation at oblique incidence and with the electric vector

${ }^{a)}$ Electronic mail: lartus@ija.csic.es. polarized parallel to the plane of incidence (TM waves). ${ }^{19,20}$ The observation of transmission minima at the frequency of LO modes (i.e., the Berreman effect) and TO modes is limited to samples that are significantly thinner than the wavelength of the electromagnetic radiation corresponding to the reststrahlen band of the material. This condition, easily fulfilled for epilayers and heterostructures produced by molecular beam epitaxy (MBE) or chemical vapor deposition, provides an alternative means to determine the frequency of both TO and LO phonons in polar compounds and structures. ${ }^{20,21}$ The transmission measurements at oblique incidence also allow one to directly measure the frequency of LO-plasmon coupled modes, from which the free-carrier concentration of the samples can be evaluated. ${ }^{22}$

The observation of transmission minima corresponding to TO or LO phonons in group-III nitrides is however limited to samples grown on substrates that are not highly light absorbing in the spectral region of interest. Epitaxial thin films and structures based on GaN or AlN have been grown on many different substrates such as sapphire, Si, or GaAs. While sapphire displays strong absorption in the $380-900 \mathrm{~cm}^{-1}$ spectral region ${ }^{23}$ and as a consequence it cannot be used for transmission experiments, the reststrahlen band of GaAs $\left(250-350 \mathrm{~cm}^{-1}\right)$ is well separated from the $A_{1}(\mathrm{TO}, \mathrm{LO})$ and $E_{1}(\mathrm{TO}, \mathrm{LO})$ phonon frequencies of $\mathrm{GaN}$ and AlN. In turn, owing to its nonpolar nature, $\mathrm{Si}$ is IR inactive and absorbs IR radiation very weakly. Hence, Si substrates are very well-suited for IR transmission studies of nitride compounds. Note that, in contrast, Si exhibits very strong first-order and second-order Raman features that may overlap with the phonon modes of the nitrides in the Raman spectra. 
TABLE I. Basic details of the GaN, AlN, and $\mathrm{Al}_{x} \mathrm{Ga}_{1-x} \mathrm{~N}$ epilayers studied in this work.

\begin{tabular}{lcccc}
\hline \hline Sample & Epilayer & Substrate & $\begin{array}{c}\text { Thickness } \\
(\mu \mathrm{m})\end{array}$ & Buffer layers \\
\hline $\mathrm{A}$ & $c$-GaN & $\mathrm{GaAs}(001)$ & 0.3 & $\mathrm{GaAs}(150 \mathrm{~nm})$ \\
$\mathrm{B}$ & $c-\mathrm{GaN}$ & $\mathrm{GaAs}(001)$ & 0.3 & $\mathrm{AlN}(100 \mathrm{~nm}) / \mathrm{GaN}(150 \mathrm{~nm}) / \mathrm{GaAs}(150 \mathrm{~nm})$ \\
$\mathrm{C}$ & $w-\mathrm{GaN}(\mathrm{Be}-\mathrm{doped})$ & $\mathrm{Si}(111)$ & 0.16 & $\ldots$ \\
$\mathrm{D}$ & $w-\mathrm{Al}_{0.02} \mathrm{Ga}_{0.98} \mathrm{~N}$ & $\mathrm{Si}(111)$ & 0.2 & $\mathrm{AlN}(100 \mathrm{~nm})$ \\
$\mathrm{E}$ & $w-\mathrm{Al}_{0.25} \mathrm{Ga}_{0.75} \mathrm{~N}$ & $\mathrm{Si}(111)$ & 0.2 & $\mathrm{AlN}(140 \mathrm{~nm})$ \\
$\mathrm{F}$ & $w-\mathrm{Al}_{0.29} \mathrm{Ga}_{0.71} \mathrm{~N}$ & $\mathrm{Si}(111)$ & 0.2 & $\cdots$ \\
$\mathrm{G}$ & $w-\mathrm{AlN}$ & $\mathrm{Si}(111)$ & 0.4 & $\cdots$ \\
\hline \hline
\end{tabular}

The aim of this work is to apply IR transmission spectroscopy to study group-III nitrides. Two types of samples are investigated: (i) cubic $(c) \mathrm{GaN}$ and $\mathrm{AlN}$ thin films grown on (100)-oriented GaAs substrates and (ii) wurtzite (w) GaN, $\mathrm{AlN}$, and $\mathrm{Al}_{x} \mathrm{Ga}_{1-x} \mathrm{~N}(x<0.3)$ thin films grown on $(111)$ oriented Si substrates. Our measurements, performed in normal and oblique incidence, allow us to detect transmission minima corresponding to the TO and LO phonons both in the cubic and in the wurtzite samples. We discuss our results in terms of the relevant electromagnetic theory of IR transmission in wurtzite thin films, which differs from the standard theory for isotropic materials. In spite of the uniaxial symmetry of wurtzite compounds, the isotropic approximation has been widely used in the literature to analyze the IR optical response of group-III nitrides. ${ }^{2,4,6,8}$

The bandgap of AlGaN is significantly higher than the energy corresponding to visible laser excitations. As a consequence, the use of visible Raman scattering to investigate structures containing very thin films of $\mathrm{AlGaN}$ (i.e., films with very small scattering volumes) may be hampered by the low Raman efficiency exhibited by this ternary alloy, in particular for large $\mathrm{Al}$ contents. In addition, given that widebandgap compounds are transparent to visible radiation, the Raman spectra may be obscured by intense peaks arising from the substrate. The Raman signal from the nitride films may be enhanced by using UV, near-resonant laser excitations. ${ }^{24}$ However, owing to absorption effects, the more involved UV Raman experiments only allow one to probe the shallowest layers of the samples. Therefore, the IR transmission measurements at oblique incidence may emerge as a complementary tool to Raman scattering for the characterization of structures based on $\mathrm{Al}(\mathrm{Ga}) \mathrm{N}$. Here we compare the IR transmission data with visible Raman-scattering measurements. We show that the IR technique may be particularly useful for the direct observation of phonon peaks from very thin layers with high $\mathrm{Al}$ content. In the case of $w$-AlGaN, the transmission spectra reveal the composition behavior of the $A_{1}(\mathrm{LO})$ and the GaN-like $E_{1}(\mathrm{TO})$ phonon modes of the ternary alloy. In the composition range investigated, the IR transmission data are in good agreement with Raman and IR reflectance results reported in the literature.

\section{EXPERIMENT}

IR transmission measurements were performed on different series of $c$-GaN, $w$-GaN, $w$-AlGaN, and $w$-AlN epilayers grown by $\mathrm{MBE}$ in different laboratories. Details of the samples such as the substrate material, presence of a buffer layer, and the composition and thickness of the epilayers can be found in Table I. All wurtzite samples were grown with the crystallographic $c$ axis parallel to the growth direction.

Room-temperature unpolarized transmission spectra were obtained with a BOMEM DA.8 rapid scanning Fourier transform IR spectrometer. The experiments were performed by using a globar source, a $\mathrm{KBr}$ beam splitter, and a $\mathrm{HgCdTe}$ [mercury cadmium telluride (MCT)] detector. The spectra were typically recorded with $1 \mathrm{~cm}^{-1}$ resolution and $100 \mathrm{co-}$ additions. The angle between the incident beam and the normal to the surface of the samples $\left(\theta_{i}\right)$ was adjusted by rotating the sample holder, which could be set to within $\sim 5^{\circ}$ of the desired angle.

Raman experiments were carried out at RT by using a confocal Raman microprobe with a $100 \times$ objective. The typical spot size was of about $1 \mu \mathrm{m}$. The spectra were recorded with a Jobin-Yvon T64000 triple-grating spectrometer equipped with a coupled charge detector cooled with liquid nitrogen. The $514.5 \mathrm{~nm}$ line of an Ar laser was used as excitation source.

\section{THEORETICAL CONSIDERATIONS}

From the point of view of far-IR spectroscopy, a polar film of thickness $d$ may be considered as thin provided that $\omega / c \gg d$, where $\omega$ is the frequency of the incident electromagnetic wave and $c$ is the speed of light. In a polar thin film, there exist two types of normal modes of vibration: (i) modes with the particle motion parallel to the plane of the film (TO modes) and (ii) modes with the particle motion perpendicular to the plane of the film (LO modes). As is well known, the long wavelength polarization fields associated to the LO modes give rise to the LO-TO splitting.

When an electromagnetic wave is incident on the film with the electric field polarized perpendicular to the plane of incidence [i.e., an $s$ wave, also known as transverse-electric (TE) wave], the radiation can only interact with the TO modes regardless of the angle of incidence $\left(\theta_{i}\right)$. In contrast, both the TO and LO modes can be excited by radiation that impinges on the film at an angle $\theta_{i}$ with the electric field polarized parallel to the plane of incidence [i.e., a $p$ wave, also known as transverse-magnetic (TM) wave]. ${ }^{19,20}$ In such case, the electric field has a component in the direction of the particle motion for both the TO and LO phonons.

While the optical response of crystals with cubic symmetry is isotropic, the response of crystals with the wurtzite 
(uniaxial) structure depends on the orientation of the crystal and on the geometry considered (i.e., optic axis in the plane of incidence or perpendicular to it). To describe the far-IR response of wurtzite crystals, two different refractive indexes for each polarization configuration (i.e., two dielectric functions) are required. Depending on the geometry considered and the angle of incidence, transmission minima corresponding to the $A_{1}(\mathrm{TO}), A_{1}(\mathrm{LO}), E_{1}(\mathrm{TO})$, or the $E_{1}(\mathrm{LO})$ modes can be detected. ${ }^{20}$

\section{A. Isotropic thin films}

For the sake of completeness, we first revisit the electromagnetic theory for far-IR transmission in an isotropic thin film (see Ref. 20 and references therein for details). In what follows, we focus on TM waves since only this radiation allows the observation of transmission minima at the frequency of LO modes. For isotropic films with complex dielectric function $\epsilon(\omega)=n^{2}(\omega)$, the fractional transmission for the TM waves is given by ${ }^{20}$

$$
T_{\mathrm{TM}}=\left|\left[\cos \kappa d-i\left(\frac{\kappa^{2}+n^{4} k^{2}}{2 n^{2} \kappa k}\right) \sin \kappa d\right]^{-1}\right|^{2},
$$

where $n(\omega)$ is the refractive index of the material, $k$ $=(\omega / c) \cos \theta_{i}$ is the wave vector component of the incident radiation parallel to the film, and $\kappa=(\omega / c)\left[n^{2}-\sin ^{2} \theta_{i}\right]^{1 / 2}$. The definition of $\kappa$ originates from the solutions of Maxwell's equations inside the film, which have the form $B_{\|}(z)$ $=a \exp ^{i \kappa z}+b \exp ^{-i \kappa z}$ for the magnetic-field component parallel to the film, where the $z$ direction is perpendicular to the plane of the film and $a$ and $b$ are constants that can be obtained with the appropriate boundary conditions.

For a binary isotropic polar compound such as $c-\mathrm{GaN}$ or $c$-AlN, the frequency-dependent refractive index is determined by the frequency of the TO and LO phonons, $\omega_{\mathrm{TO}}$ and $\omega_{\mathrm{LO}}$ :

$$
n^{2}(\omega)=\epsilon_{\infty}+\epsilon_{\infty} \frac{\omega_{\mathrm{LO}}^{2}-\omega_{\mathrm{TO}}^{2}}{\omega_{\mathrm{TO}}^{2}-\omega^{2}-i \Gamma \omega},
$$

where $\Gamma$ is the phenomenological damping coefficient for the phonon modes. The TO and LO frequencies are related to the static $\left(\epsilon_{0}\right)$ and high-frequency $\left(\epsilon_{\infty}\right)$ dielectric constants through the Lyddane-Sachs-Teller relation. If the investigated material is an isotropic two-mode ternary alloy, Eq. (2) has to be replaced by a dielectric function that includes contributions from the two polar sublattices (see Ref. 22 for details).

To illustrate the transmission model outlined above, we have plotted in Fig. 1 the calculated transmission for a TM wave at oblique incidence for $c$-AlN thin films of different thicknesses. For the calculations, we have used the phonon frequency values of $c$-AlN given in Ref. $16\left(\omega_{\mathrm{TO}}\right.$ $=655 \mathrm{~cm}^{-1}$ and $\omega_{\mathrm{LO}}=902 \mathrm{~cm}^{-1}$ ) and we have taken $\theta_{i}$ $=45^{\circ}$ and $\Gamma=10 \mathrm{~cm}^{-1}$. For the high-frequency dielectric constant, we have employed the value measured in $w$-AlN, $\epsilon_{\infty}=4.77 .{ }^{16}$ Figure 1 shows that transmission minima appear at both $\omega_{\mathrm{TO}}$ and $\omega_{\mathrm{LO}}$. For the thicker films, interference

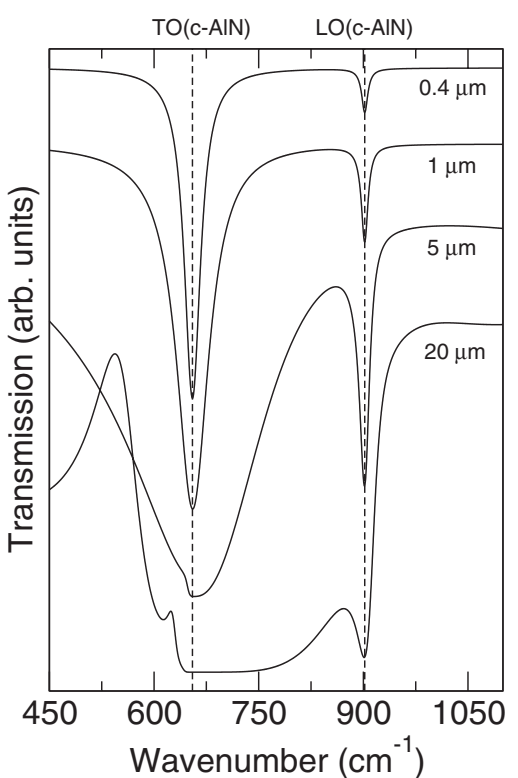

FIG. 1. Calculated far-IR transmission spectra of $c$-AIN thin films of different thicknesses, ranging from $0.4 \mu \mathrm{m}$ to $20 \mu \mathrm{m}$. Each spectrum was calculated assuming obliquely incident $\mathrm{TM}$ waves $\left(\theta_{i}=45^{\circ}\right)$.

fringes appear below the TO frequency, which results from the rapid increase in the dielectric function for $\omega$ values close to the pole at $\omega_{\mathrm{TO}}{ }^{20}$

\section{B. Uniaxial thin films}

For a uniaxial medium such as a thin film with wurtzite crystal structure, the solutions of Maxwell's equations can be found in a similar way to the isotropic case. The dielectric function, however, has to be replaced by the uniaxial dielectric tensor

$$
\epsilon(\omega)=\left(\begin{array}{ccc}
n_{\|}^{2} & 0 & 0 \\
0 & n_{\|}^{2} & 0 \\
0 & 0 & n_{\perp}^{2}
\end{array}\right),
$$

where $n_{\|}$and $n_{\perp}$ are the ordinary and extraordinary refractive indexes, respectively. The components of the dielectric tensor are given by

$$
n_{\|}^{2}(\omega)=\epsilon_{\infty \propto \|}+\epsilon_{\propto ⿻ \|} \frac{\omega_{\mathrm{LO} \|}^{2}-\omega_{\mathrm{TO} \|}^{2}}{\omega_{\mathrm{TO} \|}^{2}-\omega^{2}-i \omega \Gamma_{\|}}
$$

and

$$
n_{\perp}^{2}(\omega)=\epsilon_{\infty \perp}+\epsilon_{\infty \perp} \frac{\omega_{\mathrm{LO} \perp}^{2}-\omega_{\mathrm{TO} \perp}^{2}}{\omega_{\mathrm{TO} \perp}^{2}-\omega^{2}-i \omega \Gamma_{\perp}},
$$

where $\omega_{\mathrm{TO} \|}$ and $\omega_{\mathrm{LO} \|}$ correspond to the $E_{1}$ phonon modes of wurtzite, while $\omega_{\mathrm{TO}} \perp$ and $\omega_{\mathrm{LO} \perp}$ correspond to the $A_{1}$ modes. ${ }^{13}$ In Eqs. (4) and (5), $\Gamma_{\|, \perp}$ and $\epsilon_{\triangleright \otimes \|, \perp}$ represent the corresponding phonon damping and high-frequency dielectric constants, respectively.

For a TM wave impinging on a wurtzite thin film with the $c$-axis perpendicular to the plane of the film [see inset of Fig. 2(a)], the $z$ dependence of $B_{\|}(z)$ is the same as for the isotropic film, but with $\kappa$ given by ${ }^{25}$ 


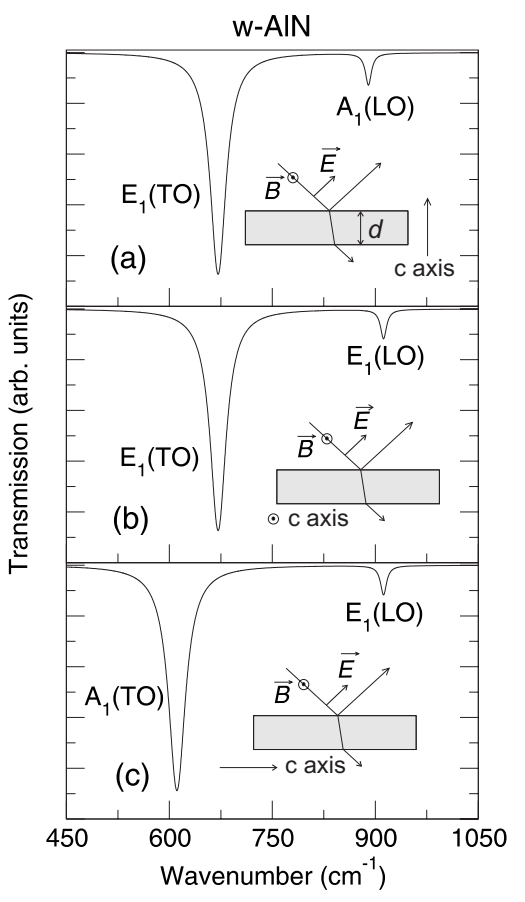

FIG. 2. Calculated far-IR transmission spectra at oblique incidence $\left(\theta_{i}\right.$ $=45^{\circ}$ ) for TM waves incident on a 400-nm-thick $w$-AlN thin film with the $c$-axis oriented (a) perpendicular to both the magnetic field of the incident radiation $(\vec{B})$ and the film surface, (b) parallel to both $\vec{B}$ and the film surface, and (c) perpendicular to $\vec{B}$ and parallel to the film surface. The insets show the different geometries considered.

$$
\kappa=(\omega / c)\left[n_{\|}^{2}-\frac{n_{\|}^{2}}{n_{\perp}^{2}} \sin ^{2} \theta_{i}\right]^{1 / 2},
$$

where $z$ is perpendicular to the plane of the film ( $z \| c$ axis). Note that, as expected, Eq. (6) reduces to the isotropic expression when $n_{\perp}=n_{\|}$. In the previous discussion, given that the $c$ axis is in the plane of incidence, the magnetic field of the incident radiation can only be polarized perpendicular to the $c$ axis. In contrast, for a TM wave impinging on a face not oriented along the $c$ axis, care has to be taken about the polarization of the magnetic field, which can be parallel or perpendicular to the optic axis [see insets of Figs. 2(b) and 2(c), respectively]. Correspondingly, the appropriate components of the dielectric tensor have to be considered in Eq. (6) for these geometries.

For a $c$-oriented thin film, similarly to the isotropic case, the solution of Maxwell's equations shows that the fractional transmission for the TM waves is given by

$$
T_{\mathrm{TM}}=\left|\left[\cos \kappa d-i\left(\frac{\kappa^{2}+n_{\|}^{4} k^{2}}{2 n_{\|}^{2} \kappa k}\right) \sin \kappa d\right]^{-1}\right|^{2},
$$

with $\kappa$ given by Eq. (6). For a film with its surface parallel to the $c$ axis, the previous expression still holds provided that the plane of incidence is perpendicular to the optic axis [inset of Fig. 2(b)], since in this case, the electric-field component of the electromagnetic radiation parallel to the plane of the film is also perpendicular to the $c$ axis. In contrast, $n_{\|}$has to be replaced by $n_{\perp}$ when the plane of incidence and the $c$ axis are parallel [inset of Fig. 2(c)], since in this case, the electric-

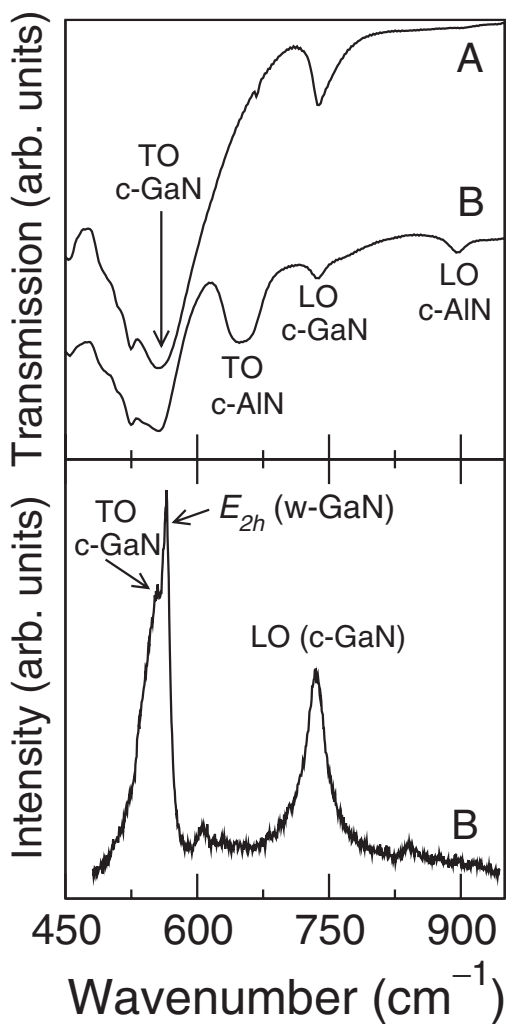

FIG. 3. (Upper panel) Far-IR transmission spectra in oblique incidence of two $c$-GaN epilayers grown on (001)-GaAs with an AlN buffer layer (curve $B$ ) and without an AlN buffer layer (curve $A$ ). (Lower panel) Visible Raman spectrum $\left(\lambda_{\mathrm{exc}}=514.5 \mathrm{~nm}\right)$ of the $c-\mathrm{GaN} / \mathrm{GaAs}$ epilayer grown with an AlN buffer layer.

field component in the plane of the film and the optic axis are parallel.

Figures 2(a)-2(c) show the calculated transmission for a TM wave at oblique incidence for a 400-nm-thick $w$-AlN thin film. The three geometries discussed above, depicted in the insets of each figure, have been considered. For the calculations, we have used the phonon frequency values of $w$-AlN given in Ref. $16 \quad\left(\omega\left[E_{1}(\mathrm{TO})\right]=670.8 \mathrm{~cm}^{-1}\right.$, $\omega\left[E_{1}(\mathrm{LO})\right]=912 \mathrm{~cm}^{-1}, \quad \omega\left[A_{1}(\mathrm{TO})\right]=611 \mathrm{~cm}^{-1}, \quad$ and $\left.\omega\left[A_{1}(\mathrm{LO})\right]=890 \mathrm{~cm}^{-1}\right)$, and we have taken $\theta_{i}=45^{\circ}, \epsilon_{\infty\|\|}$ $=\epsilon_{\infty \perp}=4.77,{ }^{16}$ and $\Gamma=10 \mathrm{~cm}^{-1}$. The figure shows that transmission minima corresponding to the normal modes of the film for each configuration, with particle motions parallel (TO) and perpendicular (LO) to the plane of the film, are expected in all the geometries for oblique incidences. In the particular case of $c$-oriented wurtzite films, these are the $E_{1}(\mathrm{TO})$ and the $\mathrm{A}_{1}(\mathrm{LO})$ modes, respectively [see Fig. 2(a)].

\section{RESULTS AND DISCUSSION}

\section{A. Cubic GaN and AIN}

The upper panel of Fig. 3 shows IR transmission spectra in oblique incidence $\left(\theta_{i} \sim 45^{\circ}\right)$ of samples $\mathrm{A}$ and $\mathrm{B}$, which consist of $0.3-\mu \mathrm{m}$-thick $c$-GaN epilayers grown on a (100)GaAs substrate (see Table I). The epilayer of sample A was grown on a $0.15-\mu \mathrm{m}$-thick GaAs buffer layer, while the epilayer of sample B was grown on a $0.15 \mu \mathrm{m} \mathrm{GaAs}$ buffer, followed by a $0.15 \mu \mathrm{m} \mathrm{GaN} \mathrm{layer} \mathrm{and} \mathrm{a} 0.10 \mu \mathrm{m}$ AlN buffer. The reststrahlen band of GaAs, in the $268-292 \mathrm{~cm}^{-1}$ 
region, does not appear in the figure because it is below the detection range of the MCT detector. The spectra of both samples display transmission dips at 555 and $742 \mathrm{~cm}^{-1}$ corresponding to the TO and LO phonon modes of $c-\mathrm{GaN}^{3}$ As predicted by theory (see Sec. III), the LO modes are not observed in the normal-incidence experiments (not shown). In addition to the GaN-related features, the spectrum of sample B displays transmission dips that can be attributed to the TO and LO modes of $c$-AlN. The positions of these two features, located at 651 and $897 \mathrm{~cm}^{-1}$, are in good agreement with the values obtained by Raman scattering in bulk crystals (655 and $902 \mathrm{~cm}^{-1}$, see Ref. 16). The lower frequency values found for the thin AlN layer of sample B in comparison to bulk samples may be related to tensile strain as a consequence of the lower lattice parameter of AlN with respect to the GaAs substrate.

Next, we compare the IR transmission data obtained from the group-III nitride cubic samples with the results of visible Raman-scattering measurements. The lower panel of Fig. 3 displays the Raman spectrum for sample B, acquired with the $514.5 \mathrm{~nm}$ line of an $\mathrm{Ar}^{+}$laser. Raman peaks corresponding to the TO and LO modes of $c$-GaN are clearly observed. An additional Raman peak at $565 \mathrm{~cm}^{-1}$, which can be attributed to the $E_{2 h}$ mode of $w$-GaN, appears in the spectrum of this sample. The presence of this peak shows that the AlN buffer promotes the growth of wurtzite domains in the $\mathrm{GaN}$ epilayer. However, as is evident from the upper panel of Fig. 3, the presence of $w-G a N$ domains in sample $\mathrm{B}$ is not detected by IR transmission because the frequency of the IR active modes (LO and TO modes) for both phases is very similar. Also, the transmission dips are much broader than the corresponding Raman peaks. In contrast, no signal from the AlN layer appears in the Raman spectra of sample B. Note that for the visible excitation wavelength used to acquire the Raman spectra $(514.5 \mathrm{~nm})$, the GaN epilayers are transparent. Thus, the absence of Raman signal from the AIN buffer can be explained by the low Raman efficiency of AlN for this wavelength and also by the relatively small thickness of the AlN buffer $(0.10 \mu \mathrm{m})$. We would like to emphasize that, given the thickness of the GaN epilayer in sample $\mathrm{B}$, the use of UV, near-resonant wavelengths would not permit one to detect the AlN layer since the excitation radiation would then be absorbed by the GaN epilayer. While the Raman measurements are clearly superior in sensitivity and resolution to study the GaN films of sample B, they fail to detect the weak modes of the AlN buffer layer contained in this sample, which can be otherwise probed unambiguously with the Berreman technique.

\section{B. Wurtzite $\mathrm{Al}(\mathrm{Ga}) \mathrm{N}$}

As discussed in Sec. III, the transmission spectrum of $c$-oriented wurtzite material is dominated by the $E_{1}(\mathrm{TO})$ mode. This can be seen in Fig. 4 for the case of an AlN thin film grown on $\mathrm{Si}(111)$ (sample $\mathrm{G}$ ). The figure shows four different spectra acquired with different incidence angles $\left(0^{\circ}\right.$, $15^{\circ}, 30^{\circ}$, and $\left.45^{\circ}\right)$. The strong $\operatorname{dip}$ at $\sim 670 \mathrm{~cm}^{-1}$ that appears in all the spectra can be assigned to the $E_{1}(\mathrm{TO})$ mode of AlN. In addition to this feature, a dip at $\sim 890 \mathrm{~cm}^{-1}$, the

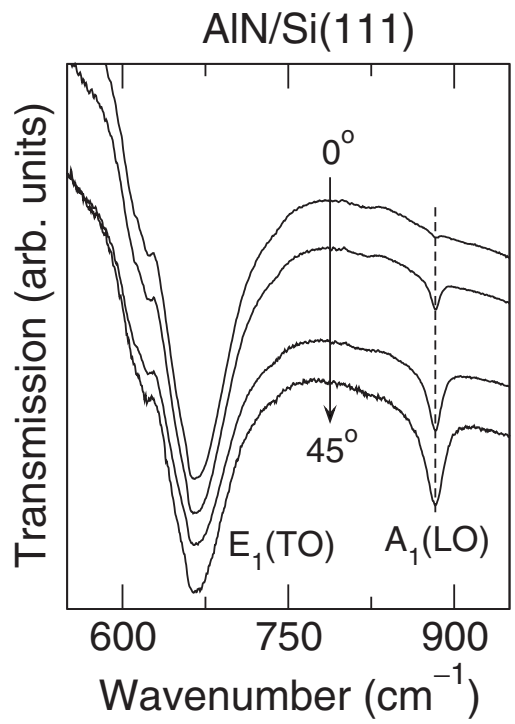

FIG. 4. IR transmission of a $w$-AlN epilayer grown by MBE on $\mathrm{Si}(111)$ (sample G) for different angles of incidence. The spectra correspond to $\theta_{i}$ $=0^{\circ}, 15^{\circ}, 30^{\circ}$, and $45^{\circ}$.

intensity of which increases with the angle of incidence of the electromagnetic radiation, is also observed. This feature corresponds to the $A_{1}(\mathrm{LO})$ mode of AlN. Owing to minor deviations from perfect normal incidence, a very weak $A_{1}$ (LO) dip is also observed in the spectrum acquired with $\sim 0^{\circ}$. More importantly, no dips corresponding to the $E_{1}(\mathrm{LO})$ mode of $w$-AlN appear in any of the spectra, in agreement with theory.

It should be borne in mind that in Raman-scattering experiments on wurtzite $\mathrm{AlN}$, an interaction between the $A_{1}$ and $E_{1}$ phonons takes place when the crystal high-symmetry axes are tilted relative to the phonon propagation direction. The resulting mixed modes, known as quasi-TO and quasi-LO modes, are shifted with respect to the pure $A_{1}$ or $E_{1}$ modes. ${ }^{26}$ As discussed in Sec. III, no mixing between the $A_{1}$ and $E_{1}$ modes occurs in the case of the oblique-incidence IR transmission experiments on samples oriented along highsymmetry axes because the symmetry of the respective TO and LO modes of the polar film (i.e., the modes with which TE and TM waves interact, respectively) is not affected by the angle of the incident radiation. Thus, only pure $A_{1}$ and $E_{1}$ modes are detected with the Berreman technique.

In Fig. 5 we show the transmission spectra at oblique incidence for three different $\mathrm{Al}_{x} \mathrm{Ga}_{1-x} \mathrm{~N}$ epilayers grown on $\mathrm{Si}(111)$ substrates (samples D-F). These epilayers were grown on an AlN buffer layer. For comparison, in Fig. 5 we have also included IR spectra of $\mathrm{GaN}$ and AlN epilayers (samples $\mathrm{C}$ and $\mathrm{G}$, respectively). As can be observed in the figure, transmission dips corresponding to the $A_{1}(\mathrm{LO})$ mode and to the GaN-like $E_{1}(\mathrm{TO})$ mode appear in all the spectra of the $\mathrm{GaN}$ and $\mathrm{AlGaN}$ epilayers. In samples $\mathrm{D}, \mathrm{E}$, and $\mathrm{F}$ we also observe the corresponding $E_{1}(\mathrm{TO})$ and $A_{1}(\mathrm{LO})$ modes arising from the AlN buffer layers. Although the $E_{1}(\mathrm{TO})$ mode appears as a fairly broad dip in the spectra of the AlGaN samples, it can be seen in the figure that this feature displays an upward frequency shift with increasing Al content. In the lower panel of Fig. 6, we plot the frequency of 


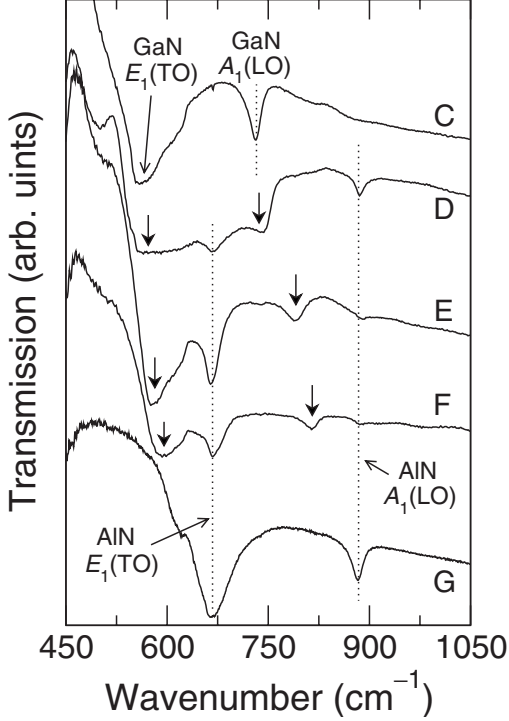

FIG. 5. IR transmission spectra of different $\mathrm{Al}_{x} \mathrm{Ga}_{1-x} \mathrm{~N} / \mathrm{AlN} / \mathrm{Si}(111)$ epilayers grown by MBE with $x=2 \%, 25 \%$, and $29 \%$ (curves $D-F$, respectively). Curves $C$ and $G$ correspond to $w-G a N$ and $w$-AlN. The vertical arrows indicate the position of the $A_{1}(\mathrm{LO})$ mode and of the GaN-like $E_{1}(\mathrm{TO})$ mode of AlGaN.

the GaN-like $E_{1}(\mathrm{TO})$ mode obtained from the transmission spectra as a function of $x$. The frequency of this mode increases monotonically with $x$, in agreement with the Raman results reported by Davydov et al. ${ }^{17}$ on hexagonal AlGaN. Note that we do not observe the AlN-like $E_{1}(\mathrm{TO})$ mode for any of our AlGaN epilayers. This is similar to the observations in Ref. 17, where no AlN-like $E_{1}(\mathrm{TO})$ mode was found in the Raman spectra of $\mathrm{Al}_{x} \mathrm{Ga}_{1-x} \mathrm{~N}$ for $\mathrm{Al}$ compositions up to $x \sim 0.6$.

On the other hand, we observe a clear, broad dip in all the spectra in Fig. 5 that can be the assigned to the $A_{1}(\mathrm{LO})$ phonon mode of AlGaN. As discussed in detail in Ref. 17, both this mode and the $E_{1}(\mathrm{LO})$ mode exhibit a one-mode behavior in this alloy system. We plot in the upper panel of Fig. 6 the composition behavior of this mode as obtained from the IR spectra. As in Ref. 17, we find that the $A_{1}(\mathrm{LO})$

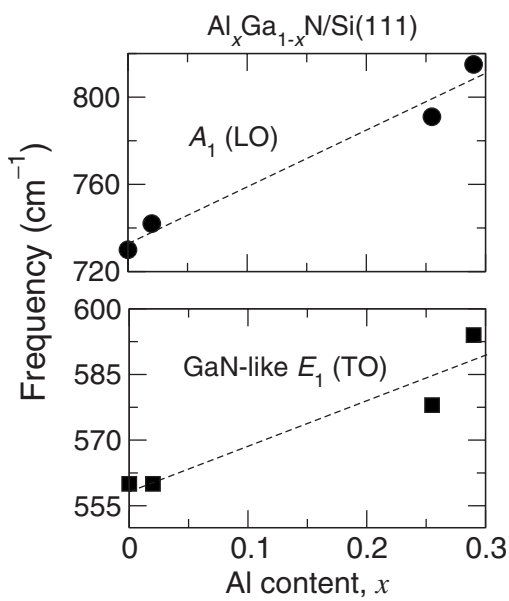

FIG. 6. Frequency behavior of the $A_{1}(\mathrm{LO})$ (upper panel) and the GaN-like $E_{1}(\mathrm{TO})$ (lower panel) phonon modes as obtained with the obliquely incident transmission measurements on the $\mathrm{Al}_{x} \mathrm{Ga}_{1-x} \mathrm{~N}$ epilayers studied in this work. The dashed lines are guides to the eye.

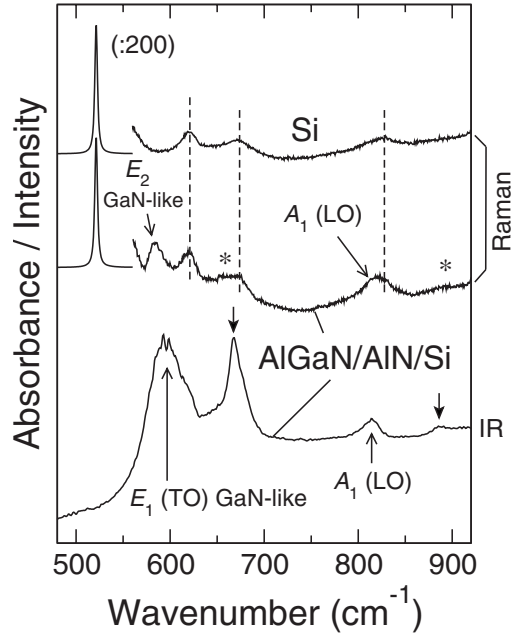

FIG. 7. Raman spectrum (middle curve) and far-IR absorbance spectrum at oblique incidence (lower curve) for an $\mathrm{Al}_{0.29} \mathrm{Ga}_{0.71} \mathrm{~N}$ epilayer grown on a $\mathrm{Si}(111)$ substrate with an AlN buffer (sample F). The upper curve shows the Raman spectrum of bulk $\mathrm{Si}(111)$. The asterisks in the Raman spectrum of the $\mathrm{Al}_{0.29} \mathrm{Ga}_{0.71} \mathrm{~N}$ epilayer indicate the position of the $E_{2 h}$ and $A_{1}(\mathrm{LO})$ modes of the AlN buffer layer. The vertical arrows in the IR spectrum refer to the $E_{1}(\mathrm{TO})$ and $A_{1}(\mathrm{LO})$ modes of the AlN buffer layer.

mode increases monotonically with increasing $x$. In the measurements performed under normal incidence, no residual signal of the $A_{1}(\mathrm{LO})$ mode was detected. As in the case of AlN, no features corresponding to the $E_{1}(\mathrm{LO})$ mode were observed in any of the oblique-incidence measurements on the $c$-oriented $\mathrm{AlGaN}$ epilayers.

Next, in order to assess the usefulness of the IR technique to study the group-III nitrides, we compare the IR transmission spectra of the AlGaN/AlN/Si(111) samples with the results of visible Raman scattering $\left(\lambda_{\mathrm{exc}}=514.5 \mathrm{~nm}\right)$. It should be first noted that the Raman efficiency of the different Raman-active phonon modes of $\mathrm{Al}_{x} \mathrm{Ga}_{1-x} \mathrm{~N}$ depends on both $x$ and on the energy of the excitation source used to perform the experiments. In other words, different laser wavelengths in the UV spectral range should be used to enhance the Raman signal of the different layers investigated. However, visible laser lines are commonly employed to study group-III nitrides. While for bulk samples or thick films the large scattering volumes involved yield strong Raman signals, weak Raman signals are expected in the case of very thin AlGaN films. In such case, the signal of the substrate material may also preclude the observation of the weakest peaks from the nitride layers.

In Fig. 7, we plot transmission and Raman spectra of one of the AlGaN/AlN/Si(111) epilayers studied in this work (sample F). For convenience, the vertical scale of the IR spectrum has been plotted as absorbance (lower curve). In the figure, we have included the Raman spectrum of bulk $\mathrm{Si}(111)$ for comparison (upper curve). In this curve, besides the strong first-order peak of $\mathrm{Si}$ at $521 \mathrm{~cm}^{-1}$, three secondorder features at about 620,670 , and $825 \mathrm{~cm}^{-1}$ are clearly visible. These three features are also detected in the Raman spectrum of sample F (intermediate curve) and overlap with some of the Raman peaks of the AlGaN and AlN layers. In particular, the $A_{1}(\mathrm{LO})$ mode of AlGaN, located at $\sim 815 \mathrm{~cm}^{-1}$, overlaps with the Si-related band at $825 \mathrm{~cm}^{-1}$, 
which might lead one to overestimate the frequency of the $A_{1}$ (LO) mode from the Raman measurements. Contrary, as discussed above, the $A_{1}(\mathrm{LO})$ mode is clearly visible in the IR oblique-incidence spectrum (see the lower curve in Fig. 7).

While in the IR spectrum of sample $F$ the GaN-like $E_{1}$ (TO) mode of AlGaN appears as a prominent feature at $\sim 595 \mathrm{~cm}^{-1}$, the nonpolar GaN-like $E_{2 h}$ mode of AlGaN can only be observed in the Raman spectrum as a weak band at $\sim 585 \mathrm{~cm}^{-1}$. For the AlN buffer only extremely weak Raman features corresponding to the $E_{2 h}$ and $A_{1}(\mathrm{LO})$ modes are detected (peaks marked with an asterisk at $\sim 650$ and $\left.\sim 890 \mathrm{~cm}^{-1}\right)$. In contrast, clear $E_{1}$ (TO) and $A_{1}(\mathrm{LO})$ bands from the AlN buffer appear in the IR spectrum (peaks marked with an arrow at $\sim 670$ and $\sim 890 \mathrm{~cm}^{-1}$ ). These results show the usefulness of the IR technique for the direct detection of phonon peaks arising from very thin layers of semiconductor compounds whose scattering efficiency is low in visible Raman spectroscopy.

\section{CONCLUSIONS}

We have performed IR transmission experiments at normal and oblique incidence in a series of GaN, AlN, and AlGaN films grown by $\mathrm{MBE}$ on either GaAs and $\mathrm{Si}(111)$ substrates. We have observed clear signatures corresponding to both the TO and the LO modes in the cubic films, grown on (001)-GaAs, and in the wurtzite samples, grown on $\mathrm{Si}(111)$. In the case of the $c$-oriented $w$-AlGaN epilayers, the $A_{1}(\mathrm{LO})$ and the GaN-like $E_{1}(\mathrm{TO})$ modes are detected. The observation of transmission dips arising from the nitride phonons is possible because both the GaAs and $\mathrm{Si}(111)$ substrates are nearly transparent in the spectral region of interest $\left(550-950 \mathrm{~cm}^{-1}\right)$. We have discussed our results in terms of the electromagnetic theory of IR transmission in cubic and wurtzite thin films. To explore the usefulness of the Berreman technique, we have compared the IR data with the results of visible Raman-scattering measurements. In the particular case of the samples grown on Si substrates, the strong first- and second-order Raman peaks of $\mathrm{Si}$ overlap with the phonon modes of the nitrides. In contrast, the Berreman technique makes it possible to unambiguously characterize the nitride films. Thus, we conclude that the IR transmission experiments at oblique incidence are particularly useful to complement Raman spectroscopy.

\section{ACKNOWLEDGMENTS}

This work was supported by the Spanish Ministry of Science and Technology (Contract No. MAT2007-63617 and the Ramon y Cajal Programme) and by the British EPSRCGB.

${ }^{1}$ A. S. Barker and M. Ilegems, Phys. Rev. B 7, 743 (1973).

${ }^{2}$ M. F. MacMillan, R. P. Devaty, and W. J. Choyke, Appl. Phys. Lett. 62, 750 (1993).

${ }^{3}$ M. Giehler, M. Ramsteiner, O. Brandt, H. Yang, and K. H. Ploog, Appl. Phys. Lett. 67, 733 (1995)

${ }^{4}$ C. Wetzel, E. E. Haller, H. Amano, and I. Akasaki, Appl. Phys. Lett. 68, 2547 (1996).

${ }^{5}$ P. Perlin, E. Litwin, B. Suchanek, W. Knap, J. Camassel, T. Suski, R. Piotrzkowski, I. Grzegory, S. Porowski, E. Kaminska, and J. C. Chervin, Appl. Phys. Lett. 68, 1114 (1996).

${ }^{6}$ G. Yu, H. Ishikawa, M. Umeno, T. Egawa, J. Watanabe, T. Soga, and T. Jimbo, Appl. Phys. Lett. 73, 1472 (1998).

${ }^{7}$ G. Mirjalili, T. J. Parker, S. Farjami, M. M. Bulbul, S. R. P. Smith, T. S. Cheng, and C. T. Foxon, Phys. Rev. B 57, 4656 (1998).

${ }^{8}$ P. Wisniewski, W. Knap, J. P. Malzac, J. Camassel, M. D. Bremser, R. F. Davis, and T. Suski, Appl. Phys. Lett. 73, 1760 (1998).

${ }^{9}$ R. Y. Krupitskaya and G. W. Auner, J. Appl. Phys. 84, 2861 (1998).

${ }^{10}$ M. Shubert, A. Kasic, T. E. Tiwald, J. Off, B. Kuhn, and F. Scholz, MRS Internet J. Nitride Semicond. Res. 4, 11 (1999).

${ }^{11}$ H. Harima, T. Inoue, S. Nakashima, H. Okumura, Y. Ishida, S. Yoshida, T. Koizumi, H. Grille, and F. Bechstedt, Appl. Phys. Lett. 74, 191 (1999).

${ }^{12}$ T. Prokofyeva, M. Seon, J. Vanbuskirk, M. Holtz, S. A. Nikishin, N. N. Faleev, H. Temkin, and S. Zollner, Phys. Rev. B 63, 125313 (2001).

${ }^{13}$ M. Holtz, T. Prokofyeva, M. Seon, K. Copeland, J. Vanbuskirk, S. Williams, S. A. Nikishin, V. Tretyakov, and H. Temkin, J. Appl. Phys. 89, 7977 (2001).

${ }^{14}$ M. Bickermann, B. M. Epelbaum, P. Heimann, Z. G. Herro, and A. Winnacker, Appl. Phys. Lett. 86, 131904 (2005).

${ }^{15}$ E. Iborra, M. Clement, L. Vergara, A. Sanz-Hervas, J. Olivares, and J. Sangrador, Appl. Phys. Lett. 88, 231901 (2006).

${ }^{16}$ H. Harima, J. Phys.: Condens. Matter 14, R967 (2002).

${ }^{17}$ V. Y. Davydov, I. N. Goncharuk, A. N. Smirnov, A. E. Nikolaev, W. V. Lundin, A. S. Usikov, A. A. Klochikhin, J. Aderhold, J. Graul, O. Semchinova, and H. Harima, Phys. Rev. B 65, 125203 (2002).

${ }^{18}$ A. Sanz-Hervas, E. Iborra, M. Clement, J. Sangrador, and M. Aguilar, Diamond Relat. Mater. 12, 1186 (2003).

${ }^{19}$ D. W. Berreman, Phys. Rev. 130, 2193 (1963).

${ }^{20}$ M. D. Sciacca, A. J. Mayur, E. Oh, A. K. Ramdas, S. Rodriguez, J. K. Furdyna, M. R. Melloch, C. P. Beetz, and W. S. Yoo, Phys. Rev. B 51, 7744 (1995)

${ }^{21}$ M. Aigle, H. Pascher, H. Kim, E. Tarhan, A. J. Mayur, M. D. Sciacca, A. K. Ramdas, G. Springholz, and G. Bauer, Phys. Rev. B 64, 035316 (2001).

${ }^{22}$ J. Ibáñez, E. Tarhan, A. K. Ramdas, S. Hernández, R. Cuscó, L. Artús, M. R. Melloch, and M. Hopkinson, Phys. Rev. B 69, 075314 (2004).

${ }^{23}$ A. S. Barker, Phys. Rev. 132, 1474 (1963).

${ }^{24}$ D. Pastor, J. Ibáñez, R. Cuscó, L. Artús, G. González-Díaz, and E. Calleja, Semicond. Sci. Technol. 22, 70 (2007).

${ }^{25}$ T. Dumelow and D. R. Tilley, J. Opt. Soc. Am. A 10, 633 (1993).

${ }^{26}$ L. Bergman, M. Dutta, C. Balkas, R. F. Davis, J. A. Christman, D. Alexson, and R. J. Nemanich, J. Appl. Phys. 85, 3535 (1999). 